DOI:10.12731/2227-930X-2017-1-18-27

\title{
DEVELOPING INCIDENT DETECTION ALGORITHM BASED ON THE MAMDANI FUZZY INFERENCE ALGORITHM
}

\section{Nikolaev A.B., Sapego Yu.S.}

Application of fuzzy logic in the incident detection system allows making a decision under uncertainty. The phase of incident detection is a process of finding difficulties in traffic. The difficulty in traffic is the main sign that there was a road accident and requires a reaction for its elimination. This leads to the use of input data that must be relevant to the vehicles and the road. These data must be considered together, and should be compared with the corresponding values for further analysis. The main parameters of the traffic flow, which can characterize its current state, are a flow rate, a volume flow.

Necessary to analyze the input data received from the sensors. After processing the input data, using the previously entered fuzzy rules, will be taken action that will improve the situation in traffic or at least not allow it worse.

Keywords: Mamdani fuzzy inference algorithm; incident detection system.

\section{Introduction}

Components of the system based on fuzzy logic can be implemented by various methods. Lotfi Zadeh developed the idea of the formalization of fuzzy control algorithm using the logical rules [16, p. 338]. For obtaining output of vaguely formulated data can use logical rules with vague predicates. Consider more detail the algorithm Mamdani fuzzy inference that will be used in this paper, as the most useful for the implementation of fuzzy control systems.

Mamdani algorithm describes several sequential steps. Each successive stage receives input values obtained in the previous step (fig. 1): 




Fig. 1. Phases of Mamdani algorithm

Consider in more detail each of the steps on the example of incident detection system, further linguistic parameters will be introduced.

\section{Determining of linguistic variables}

Linguistic variables are input and output variables of the fuzzy system. Linguistic variables consist of words or sentences of a natural language, which allows to express certain conditions and to understand them without the need for measurement or calculation to make a definite conclusion, for example, "on the section of the road was filled with" instead of "on the section of the road was 100 cars."

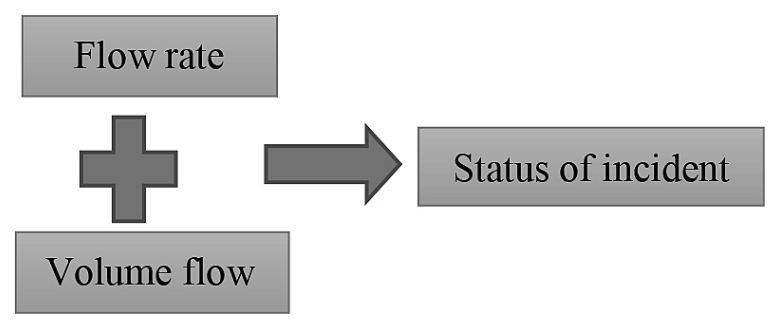

Fig. 2. The input and output linguistic variables

The next linguistic variables in incident detection system will be used to determine the occurrence of incidents (fig. 2):

- Flow Rate $=\{$ small $($ SM_SP $)$, medium $($ ME_SP), large (LA_SP) $\}$

On sections of road where the accident occurred the rate of traffic flow will certainly be slower than in an area where nothing prevents movement. Therefore this variable is necessary for the determining the occurrence of the incident. 
- Volume flow $=\left\{\operatorname{small}\left(\mathrm{SM}_{-} \mathrm{V}\right), \operatorname{medium}\left(\mathrm{ME} \_\mathrm{V}\right)\right.$, large $\left.\left(\mathrm{LA} \_\mathrm{V}\right)\right\}$

The volume flow is the number of vehicles crossing the road section in a predetermined unit of time. If the incident occurred on the road, the number of cars is less than in free motion. It should be noted pattern of volume on flow rate: if the vehicle speed is high and the volume is small, it is considered that the road is free. If the volume of vehicles remains unchanged, the flow rate drops, it means that traffic incident probability occurred.

- Status of incident $=$ \{false, true $\}$

This variable has two values: "false" - no incident, "true" - incident occurred.

Each specified linguistic variable measures certain traffic conditions; with these conditions form the rules governed the system. Stage of the determination of linguistic variables is an important step because they effect on the efficiency of the system. These variables must be translated into the fuzzy controller by using membership functions; therefore they should be defined for the above variables.

\section{The formation of fuzzy rule base}

When an incident occurs, it is formed on the road a congestion. As soon as incident is considered to be cleaned, the road capacity is increased and congestion dissipates. The algorithm determines the state of traffic changes according to the volume and rate of flow.

Define fuzzy rules that are used to describe the relationship between inputs and outputs data, as the current state of road can determine using fuzzy rules. As a result received 9 rules (Table 1):

Table 1 .

Fuzzy rules for determining the current status of the road

\begin{tabular}{|c|c|c|c|}
\hline № & Flow Rate & Volume flow & Status of incident \\
\hline 1 & \multirow{3}{*}{ Small (SM_SP) } & Small (SM_V) & true \\
\cline { 4 - 4 } & & Medium (SM_SP) & true \\
\cline { 3 - 4 } 3 & & Large (LA_SP) & true \\
\hline 4 & \multirow{3}{*}{ Medium (ME_SP) } & Small (SM_V) & true \\
\cline { 4 - 4 } & & Medium (SM_SP) & false \\
\cline { 3 - 4 } & & Large (LA_SP) & false \\
\hline
\end{tabular}


End of the table

\begin{tabular}{|l|l|c|c|}
\hline 7 & \multirow{3}{*}{ Large (LA_SP) } & Small (SM_V) & false \\
\cline { 4 - 4 } & & Medium (SM_SP) & false \\
\cline { 3 - 4 } & & Large (LA_SP) & false \\
\hline
\end{tabular}

The algorithm of incident detection will issue one of the following results:

1. Normal traffic

2. Probability of occurrence of the incident

3. Incident is detected

During a certain period of time traffic conditions will be analyzed for occurrence of the incident. If the analysis shows that the movement is not normal, it is considered «likely the incident occurred» (situation 2) or - a situation 1 . If during 3 time periods measured traffic is not normal, it is considered that an incident occurred on the road, then the output will be situation 3 . If the flow is returned to normal, it means that the incident was resolved, and the output will be situation 1 again.

\section{The fuzzification}

A characteristic of a fuzzy set is membership function, which is responsible for the process of fuzzification necessary to compensate for the lack of inaccurate input data from sensors because the equipment cannot provide reliable indication for various reasons.

For term-sets certain linguistic variables that are uncertainty such as "is in the range", should be used trapezoidal membership function (Fig. 3):

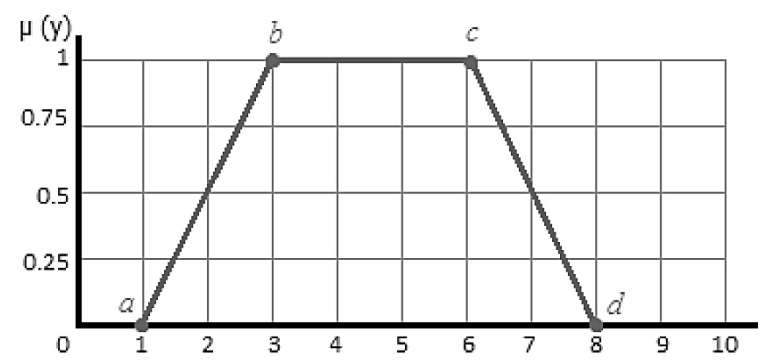

Fig. 3. An example of a trapezoidal membership function 
The trapezoidal membership function, in general, can be defined analytically by the following expression:

$$
f(x, a, b, c, d)=\left\{\begin{array}{c}
0, x \leq a \\
\frac{x-a}{b-a}, a \leq x \leq b \\
1, b \leq x \leq c \\
\frac{d-x}{d-c}, c \leq x \leq d \\
0, d \leq x
\end{array}\right\}
$$

where $a, b, c, d$-some numerical parameters that take arbitrary real values and the ordered relationship: $a \leq b \leq c \leq d$. The parameters $a$ and $d$ describe the lower base of the trapezoid, and the parameters $b$ and $d$-the upper. Furthermore, this membership function generates a normal convex fuzzy set with the characteristics: interval $(a, d)$, boundaries $(a, b)(c, d)$, core $[b, c]$.

Define the membership functions for the linguistic variable:

- Flow rate (Fig. 4):

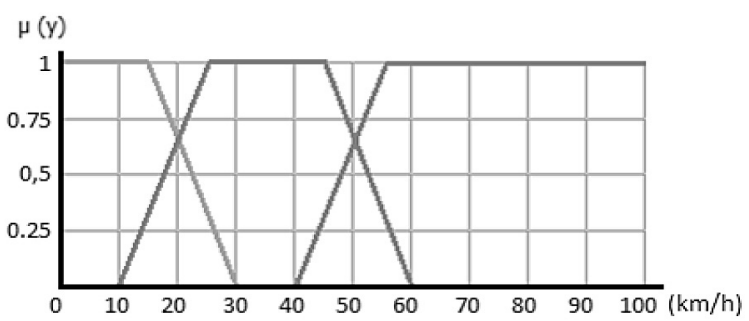

Fig. 4. Membership function for the characteristic "flow rate", where 1 - small (SM_SP), 2 -medium (ME_SP), 3 - large (LA_SP)

The value "small":

$$
\mu_{\mathrm{sm}}(S P)=\left\{\begin{array}{c}
1, S P \leq 15 \\
\frac{30-S P}{15}, 15 \leq S P \leq 30
\end{array}\right\}
$$

The value "medium":

$$
\mu_{\mathrm{me}}(S P)=\left\{\begin{array}{c}
\frac{S P-10}{15}, 10 \leq S P \leq 25 \\
1,25 \leq S P \leq 45 \\
\frac{60-S P}{15}, 45 \leq S P \leq 60
\end{array}\right\}
$$

The value "large": 


$$
\mu_{\mathrm{la}}(S P)=\left\{\begin{array}{c}
\frac{S P-40}{15}, 40 \leq S P \leq 55 \\
1,55 \leq S P
\end{array}\right\}
$$

- Volume flow (Fig. 5):

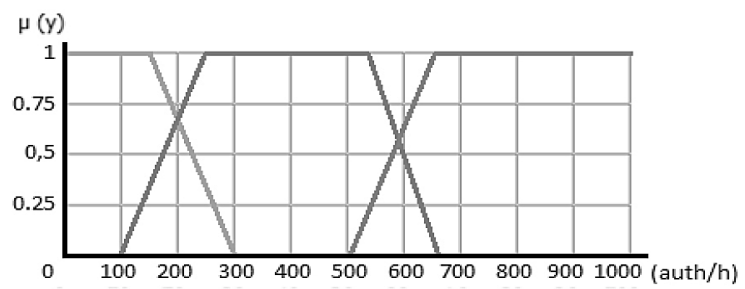

Fig. 5. Membership function for the characteristic "volume flow", where 1 - small (SM_V), 2 - medium (ME_V), 3 - large (LA_V)

The value "small":

$$
\mu_{\mathrm{sm}}(V)=\left\{\begin{array}{c}
1, \quad V \leq 250 \\
\frac{300-V}{150}, 150 \leq V \leq 300
\end{array}\right\}
$$

The value "medium":

$$
\mu_{\mathrm{me}}(V)=\left\{\begin{array}{c}
\frac{V-100}{150}, 100 \leq V \leq 250 \\
1,250 \leq V \leq 550 \\
\frac{650-V}{100}, 550 \leq V \leq 650
\end{array}\right\}
$$

The value "large":

$$
\mu_{\mathrm{la}}(V)=\left\{\begin{array}{c}
\frac{V-500}{150}, 500 \leq V \leq 650 \\
1,650 \leq V
\end{array}\right\}
$$

\section{Fuzzy inference}

Fuzzy inference consists of three main stages [3, p. 333]:

- Aggregation

- Activation

- Accumulation

Aggregation is a procedure for determining the degree of truth of $a_{i}$ conditions for each fuzzy rules from inference system. If the condition of fuzzy rule is a simple fuzzy statement, the degree of its truth corresponds to the value of the membership function corresponding to the term of a linguistic variable. If the condition is a composite statement 
(such as incident detection system), the degree of truth of a complex statement is determined based on the known values of the truth of its component elementary statements using fuzzy logic operations previously introduced in one of the pre-specified bases. For incident detection systems, it will be determined as follows:

$$
a_{i}=\min \left\{\mu_{i 1}\left(S P_{i}\right), \mu_{i 2}\left(V_{i}\right)\right\}
$$

where $\mu_{i 1}$-membership function for the characteristic «flow rate» (SP).

$\mu_{i 2}-$ membership function for the characteristic «volume flow» $(\mathrm{V})$.

$i=(1 . . n)-$ number of fuzzy rules.

Activation is the process of finding the degree of truth of each the sub-conclusion of the fuzzy rules. If the conclusion of fuzzy rule is simple fuzzy statement, the degree of its truth is algebraic multiplication of weight and the degree of the truth of the antecedent of the fuzzy rules.

If the conclusion is a composite statement, the truth degree of each of the elementary statements is algebraic multiplication of weight and the degree of the truth of the antecedent of the present fuzzy rules. If weight coefficients are not specified explicitly in the stage of forming the basis of the rules, their default values equal 1 .

Membership functions $\mu(y)$ of each elementary sub-conclusions consequent all fuzzy rules are determined by using the method of fuzzy composition - min-activation:

$$
\mu_{\text {acti }}(S P, V)=\min \left\{a_{i}, \mu_{i 1}(S P), \mu_{i 2}\left(V_{i}\right)\right\}
$$

Accumulation is the procedure of finding the membership function for each output linguistic variables. The aim of this stage is obtaining a fuzzy set (and their associations) for each output variables. Association membership functions of all sub-conclusion carried out by using max-associations method:

$$
\mu_{\text {acc }}=\max \left\{\mu_{\text {acti }}(S P, V) \ldots \mu_{\text {actn }}(S P, V)\right\}
$$

where $\mathrm{n} \in \mathrm{N}$ - total number of fuzzy rules.

The final stage is the defuzzification: process of transition from the membership functions of the output linguistic variable to its crisp (numeric) value. This phase in the developed system of incident detection is not required, as the incident detection algorithm returns a boolean value (true or false). 


\section{Implementation of the developed algorithm}

Example of system that described natural language and has two input and one output variables. If the flow rate is high and the volume flow is medium, it is considered that incident didn't occur on the road. Membership function of these linguistic variables are calculated and formed by using a fuzzy model. The final stage is operation of defuzzification operation that produces a crisp output action.

Let monitoring system returned following value of parameters: flow rate (SP) $-47 \mathrm{~km} / \mathrm{h}$, volume flow $(\mathrm{V})-565 \mathrm{auth} / \mathrm{h}$. Calculate for each membership function values according to the formulas 2-7:

Flow rate:

Volume flow:

$$
\begin{gathered}
\mu_{s m}(47)=0 \\
\mu_{m e}(47)=0,86 \\
\mu_{l a}(47)=0,47
\end{gathered}
$$

$$
\begin{gathered}
\mu_{s m}(565)=0 \\
\boldsymbol{\mu}_{\boldsymbol{m e}}(\mathbf{5 6 5})=\mathbf{0 , 8 5} \\
\mu_{l a}(565)=0,43
\end{gathered}
$$

Further, it is necessary to determine the degree of membership for each rules of the fuzzy inference system. According to the above-calculated values of membership functions for each fuzzy rule receives an activation of sub-conclusion by using min-activation method (Table 2):

Table 2.

Degree of membership for each fuzzy rule

\begin{tabular}{|c|c|c|c|}
\hline Rate & $\boldsymbol{\mu}_{s m}(\mathbf{5 6 5})$ & $\boldsymbol{\mu}_{m e}(\mathbf{5 6 5})$ & $\boldsymbol{\mu}_{l a}(\mathbf{5 6 5})$ \\
\hline $\boldsymbol{\mu}_{s m}(\mathbf{4 7})$ & $\min \{0 ; 0\}=0$ & $\min \{0 ; 0,85\}=0$ & $\min \{0 ; 0,43\}=0$ \\
\hline $\boldsymbol{\mu}_{m e}(\mathbf{4 7})$ & $\min \{0,86 ; 0\}=0$ & $\min \{0,86 ; 0,85\}=0,85$ & $\min \{0,86 ; 0,43\}=0,43$ \\
\hline $\boldsymbol{\mu}_{l a}(\mathbf{4 7})$ & $\min \{0,47 ; 0\}=0$ & $\min \{0,47 ; 0,85\}=0,47$ & $\min \{0,47 ; 0,43\}=0,43$ \\
\hline
\end{tabular}

The final step in the fuzzy inference is a stage of accumulation, on that receive fuzzy set (or association) for each of the output variables by using max-associations method:

$$
\mu=\max \{0 ; 0 ; 0 ; 0 ; 0,85 ; 0,47 ; 0 ; 0,43 ; 0,43\}
$$


Get the total value 0.85 for the fuzzy rule: «rate $=$ medium» and «volume $=$ medium». According to the table 1 the system displays the status of the incident «false», which means that the incident did not appear on the investigated section of the road.

\section{Conclusion}

In this paper, fuzzy algorithm of Mamdani was discussed in detail: defined linguistic variables, formed fuzzy rule base, offered fuzzy inference algorithm, as well as illustrates an example of the implementation of the described algorithm for incident detection system.

\section{References}

1. Abdulrahman Alkandari. Accident Detection and Action System Using Fuzzy Logic Theory // Proceedings of 2013 International Conference on Fuzzy Theory and Its Application. Dec. 6-8, 2013. Taipei, Taiwan, pp. 385-390.

2. Deniz O., Celikoglu H.B. Overview to some existing incident detection algorithms: a comparative evaluation // Procedia - Social and Behavioral Sciences. 2011, pp. 1-13.

3. Iancu I. A Mamdani Type Fuzzy Logic Controller // Fuzzy Logic Controls, Concepts, Theories and Applications. University of Craiova Romania, pp. 325-350.

4. Manstetten D., Maichle J. Determination of traffic characteristics using fuzzy logic. $11 \mathrm{pp}$.

5. Hi-ri-o-tappa K., Likitkhajorn C., Poolsawat A., Thajchayapong S. Traffic incident detection system using series of point detectors // Intelligent Transportation Systems (ITSC), 15th International IEEE Conference on. 2012, pp. 182-187.

6. Hourdos J., Garg V., Michalopoulos P. Accident Prevention Based on Automatic Detection of Accident Prone Traffic Conditions: Phase I. Final Report. CTS 08-12. 2008. 1-152 p.

7. Hyung Jin Kim, Ph.D., Hoi-Kyun Choi, Ph.D. A comparative analysis of incident service time on urban freeways // IATSS Research. Vol.25 No.1, 2001, pp. $62-72$. 
8. Mingwei Hu, Hao Tang. Development of the Real-time Evaluation and Decision Support System for Incident Management. IEEE. 2003, pp. 426-431.

9. Parkany E. A Complete Review of Incident Detection Algorithms \& Their Deployment: What Works and What Doesn't. Feb. 7, 2005. 112 p.

10. Rossi R., Gastaldi M. Fuzzy logic-based incident detection system using loop detectors data // Transportation Research Procedia 10 ( 2015), pp. 266-275.

11. Rubanov V.G., Filatov A.G., Rybin I.A. Intelligent automatic control system. Fuzzy control in technical systems. Electronic manual. URL: http://nrsu.bstu.ru/chap27.html

12. Sergio Mitrovich, Gaetano Valenti, Massimo Mancini. A decision support system (DSS) for traffic incident management in roadway tunnel infrasrtucture // Association for European Transport and contributors. 2006.

13. Viswanathan M., Lee S.H., Yang Y.K. Neuro-fuzzy Learning for Automated Incident Detection / Advances in Applied Artificial Intelligence. Volume 4031 of the series Lecture Notes in Computer Science, pp. 889-897.

14. Xie Binglei, Hu Zheng, Ma Hongwei. Fuzzy-logic-Bbased traffic incident detection algorithm for freeways // Proceedings of the Seventh International Conference on Machine Learning and Cybernetics. July 12-15, 2008, pp. 1254-1259.

15.Zadeh L.A. Fuzzy algorithms//Information and Control 12 (2). 1968, pp. 94-102.

16. Zadeh L.A. Fuzzy Sets // Information and control (8). 1965, pp. 338-353.

\section{DATA ABOUT THE AUTHORS}

Nikolaev Andrey Borisovich, Honoris Causa, Doctor of Technical Sciences, Professor, Head of Department

State Technical University - MADI

64, Leningradsky prospekt, Moscow, 125319, Russian Federation nikolaev.madi@mail.ru

Sapego Yuliya Sergeevna, Postgraduate Student, Department of Automated Control Systems

State Technical University - MADI

64, Leningradsky prospekt, Moscow, 125319, Russian Federation kafedra@asu.madi.ru 